\title{
A MICROASSEMBLED LARGE-DEFLECTION TIP/TILT MICROMIRROR FROM A SINGLE-MASK DRIE PROCESS
}

\author{
Matthew E. Last, V. Subramaniam, and Kristofer S.J. Pister \\ Berkeley Sensor and Actuator Center, University of California Berkeley \\ Berkeley, California USA
}

\begin{abstract}
An electrostatically-actuated micromirror optimized for large static pointing angles is designed, fabricated, and tested. A singlemask SOI process is used to create the actuators, mechanisms, and microassembly tools described in this paper. Two pick-and-place microassembly steps are used to (a) attach a mechanism that converts the actuators' in-plane motion into rotational motion, and (b) attach a mirror face sheet to the rotation mechanism. We report the largest static range of deflection angles for a micromirror: 0$28.2^{\circ}$ and $0-14.9^{\circ}$ at $50 \mathrm{~V}$ for each axis. Compared to the previous record [1], this corresponds to a $27 \%$ improvement in one axis but a $12 \%$ reduction in the other axis. Moreover, we use a single-mask process as opposed to the seven-mask process in [1]. Other new accomplishments reported here include a microfabricated tweezer that grabs and simultaneously rotates parts out of plane, a flexurally-suspended Zero Insertion Force (ZIF) socket for the assembled part, and a new tether design that holds the part while the tether is being broken.
\end{abstract}

\section{INTRODUCTION}

Complex actuated micromechanical systems capable of out-of-plane motion have numerous applications in fields like optics and micro-robotics. Such systems can be built in complex multi-layer MEMS processes as demonstrated in [2, 3]. There is however a tradeoff available between process complexity (difficulty of wafer-level fabrication) and postprocess complexity (chip-level operations); microassembly techniques can be used to shift this complexity from the fabrication to the post-process. Microassembly of MEMS structures using serial pick and place or various parallel directed assembly techniques has been used to integrate electronics with micromechanisms [4] and to construct electromechanical structures $[5,6]$.
Serial pick and place microassembly of surfacemicromachined parts using specially designed robotic arms with passive grippers has been demonstrated [7]. Complex assembled SOI structures for applications such as miniaturized scanning electron microscopes and variable optical attenuators $[8,9]$ have also been demonstrated. However, neither of the groups mentioned here have quantified the mechanical stability of their connectors against actuation forces. The functionality of the micromirror presented here hinges on advances made in our lab in mechanically rigid sockets. Rotation stages are fabricated in a single mask SOI process, then picked up and assembled into sockets attached to electrostatic actuators (fig 1). Linear motion of the actuators is converted to out-of-plane two-axis motion of the rotation stage. A mirror face-plate is then assembled on the rotation stage. Thus we demonstrate the use of pick and place assembly to create a high performance two-axis micromirror.

\section{FABRICATION}

The process used here and in [10] consists of a singlemask, high-aspect-ratio, deep reactive ion etch (DRIE) through the device layer of a custom-made SOI wafer. The wafer consists of a $20 \mu \mathrm{m}$ thick device layer, a $5 \mu \mathrm{m}$ buried oxide layer, and a $300 \mu \mathrm{m}$ handle wafer. A timed HF acid etch and critical point drying step release the parts from the handle wafer. A self assembled monolayer of an organosilane (FDTS) is deposited using a molecular vapor deposition system from Applied Microstructures. This serves to make the exposed silicon surfaces hydrophobic and reduces stiction between parts. An entire design cycle consisting of design changes, mask fabrication, part fabrication and assembly has been demonstrated in as little as 30 hours.


Figure 1: 2-axis rotation stage. Left: electron micrograph shows a birds-eye view of comb drive actuators, moving clamps, and assembled rotation stage. Right: SEM shows a zoomed perspective 

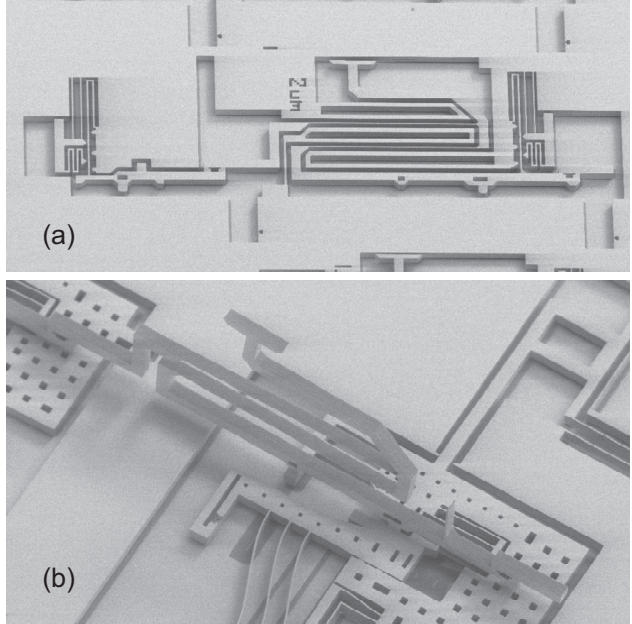

Figure 2: Rotation stage. (a) As fabricated (b) As assembled

\section{MICROASSEMBLY}

Pick and place microassembly builds complex out-of-plane mechanisms by picking up planar micro-parts, rotating them $90^{\circ}$ out-of-plane, and permanently affixing them to the SOI device layer using microfabricated sockets (fig 2). This process, along with the design of the microparts and tooling required, is described in [10]. Some modifications to the process which enabled the twoaxis micromirror are presented below.

The assembly process requires a tool that can reliably grasp a part, perform the out-of-plane rotation, and let go of it. A new springloaded rotation stage, the sidewall-gripping "ortho-gripper", is presented here (fig 3). Fabricated using the same single-mask process, it consists of two single degree-of-freedom fingers that grasp a part and rotate it exactly $90^{\circ}$. The two fingers can each move orthogonally to one another with one degree of freedom. This tool differs from previous versions of the ortho-gripper [10] in that it grips the sidewalls of the parts to be assembled instead of their top and bottom surfaces, thus SOI wafers with arbitrary buried oxide thicknesses can now be used. The analysis and design of spring-loaded ortho-grippers are shown in [11]. Operation of the sidewall-gripping version of the tool is presented here for the first time.

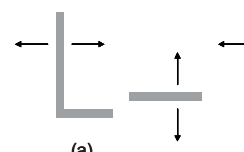

(a)

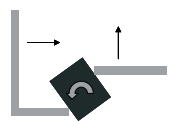

(c)

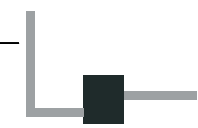

(b)

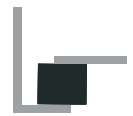

(d)

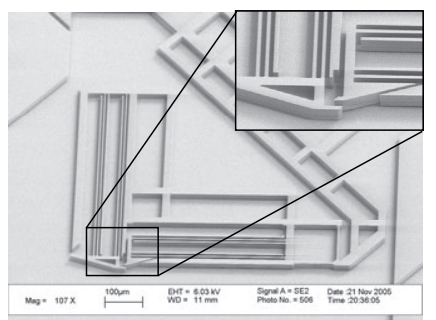

(e)
Figure 3: Ortho-gripper Principle of Operation: The fingers are shown initially grasping a part (b), in the process of rotating the part (c), and after rotation is complete (d). SEM image of sidewall-gripping Ortho-gripper (e). Detail of gripper fingers (inset)
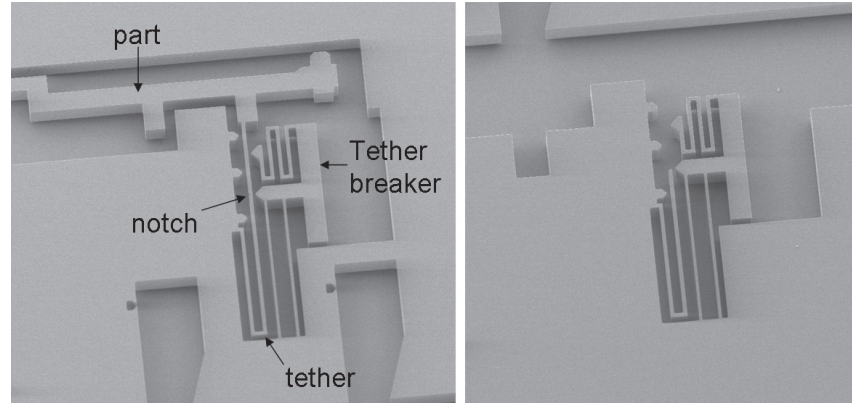

Figure 4: Tether with integrated tether-breaker

The ability of the rotation stage to reliably rotate microparts depends on the predictable breakage of the tethers that hold the part to the wafer during fabrication. A new tether design with an integrated tether-cleaving tool is presented (fig 4). This tool holds the part in place while the tether is broken, preventing the part from being flung away as the strain energy stored in the tether is released. To break the tether, the tether breaker is pushed with a probe tip. The tip of the breaker and the tip at the end of the serpentine spring touch the tether at the same time. The springloaded tip holds the part in place while the tether is being broken and prevents the part from flying away.

Finally, a fully-suspended ZIF socket with a friction-based clamping mechanism that robustly secures the assembled part is presented (fig 5). Interferometer data shows that no part of the socket comes into contact with the substrate even when experiencing reaction forces from the assembled structure. This socket provides a rigid coupling between the assembled mechanism and in-plane actuators and suspensions, without the use of sliding contacts. In addition, rigid sockets improve assembly yield by securing the part to the device layer with approximately 4 orders of magnitude more force than the ortho-gripper can exert. This is important because reliable ungrasping of the part after assembly depends on this ratio of forces. This rigidity is also useful for ensuring the shock tolerance of the assembled structure.

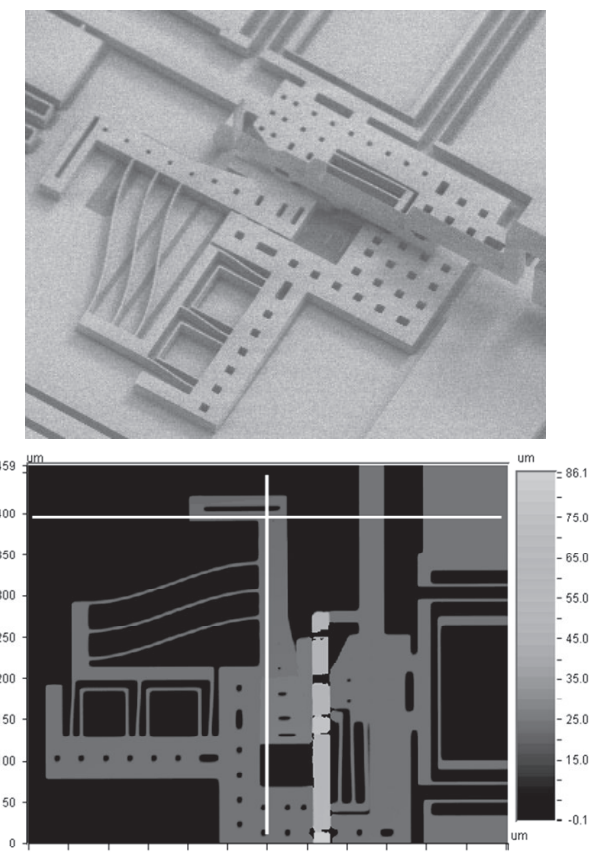

Figure 5: SEM and optical surface profile (from intereferometer) of part inserted into moving socket 


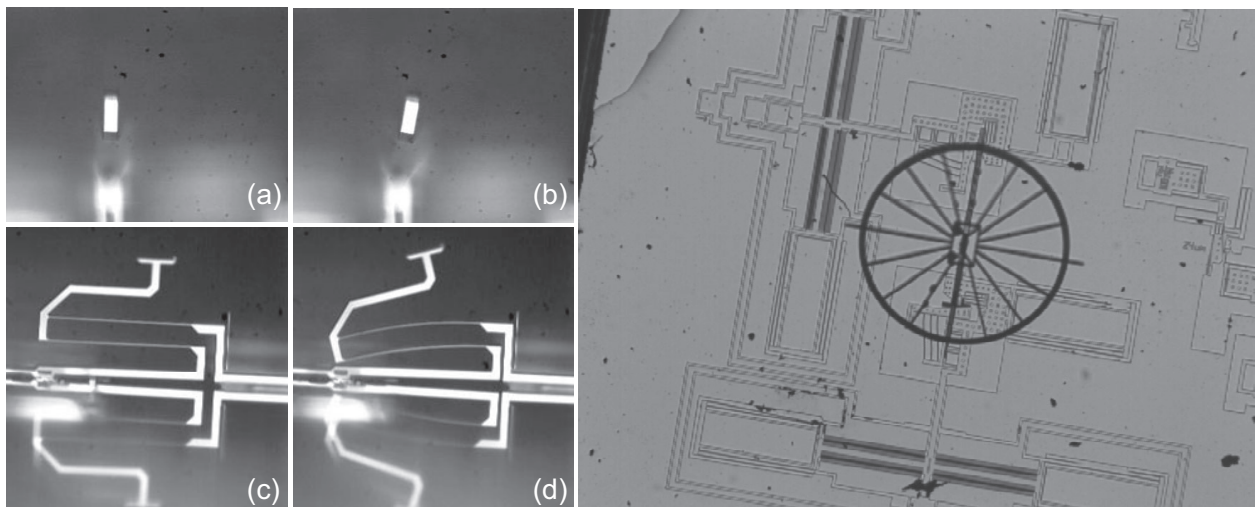

Figure 6: Left: Photomicrographs show an end-on view of motion about the torsion axis $(a, b)$ and a side view of motion about the flexion axis (c,d) Right: Optical micrograph showing assembled dummy mirror

A pull-out force $>12 \mathrm{mN}$ in each axis is required to dislodge the assembled part, corresponding to an acceleration of over 40,000g's.

\section{MICROMIRROR}

The micromirror is composed of three sub-parts: the actuators, the assembled rotation mechanism, and the assembled mirror face sheet. Two bi-directional electrostatic comb drive actuators are oriented orthogonally to one another and move in the plane of the chip. These actuators each apply $21 \mu \mathrm{N}$ at $55 \mathrm{~V}$ and provide displacements up to $17 \mu \mathrm{m}$. At high voltages, one direction of the comb drive actuator short-circuits to the substrate so only one quarter of the full range of motion of the micromirror was tested. Each actuator is attached to a flexurally-suspended socket, into which the rotation mechanism is assembled. This rotation mechanism consists of two thin parallel flexures connected at one end with a rigid beam (fig 7) which is attached to the mounting point for the mirror face sheet. The other ends of the flexures are attached to the actuators (fig 1). When actuated, the flexures either bend (flexion) or twist (torsion), resulting in the two axes of motion (figs 6). The assembled rotation mechanism thus eliminates the additional structural layers from the wafer-level fabrication process that are typically required for 2 -axis rotation stages. The two-axis rotation stage is designed to maximize DC deflection while ensuring that the stress developed on the beams is below the stress limit of silicon. A simplified rotation stage was also simulated in ANSYS (fig 7) using its non-linear modeling capabilities to yield the maximum stresses generated and the maximum angle of deflection.

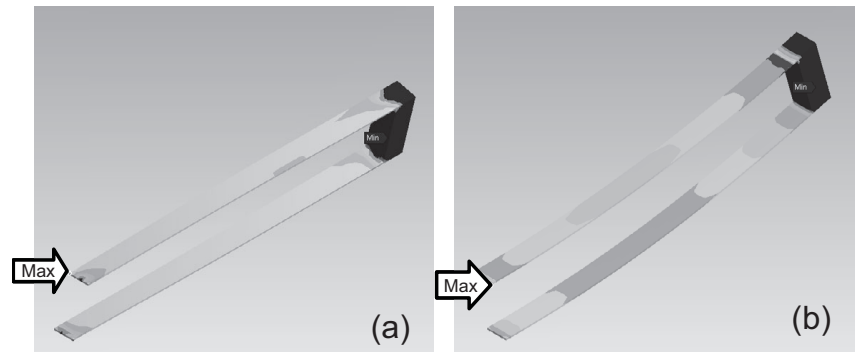

Figure 7: Simulations of 2-axis rotation stage. Von Mises stress obtained at maximum deflection in (a) torsion mode, (b) flexion mode. Maximum Stress $=200 \mathrm{MPa}$ in (a) and 100MPa in (b)
Optical deflection angle of a laser beam bounced off of the top of the rotation stage was measured to quantify the DC rotation of the devices. Mechanical rotation angle is half the optical angle shown here. Two devices were tested: Device one had two functional actuators (pull-mode about torsion and flex axes) and was tested by bouncing the laser beam off of the top of the device. The second device had only one functional axis (torsion) and was tested using a video camera (as in fig 6). This was done by using an optical microscope to look through a $45^{\circ}$ mirror placed next to the chip. Maximum DC deflections of $28^{\circ}$ (torsion) and $22^{\circ}$ (flexion) were obtained from two separate devices (fig 8).

Angle vs voltage (pull actuators)

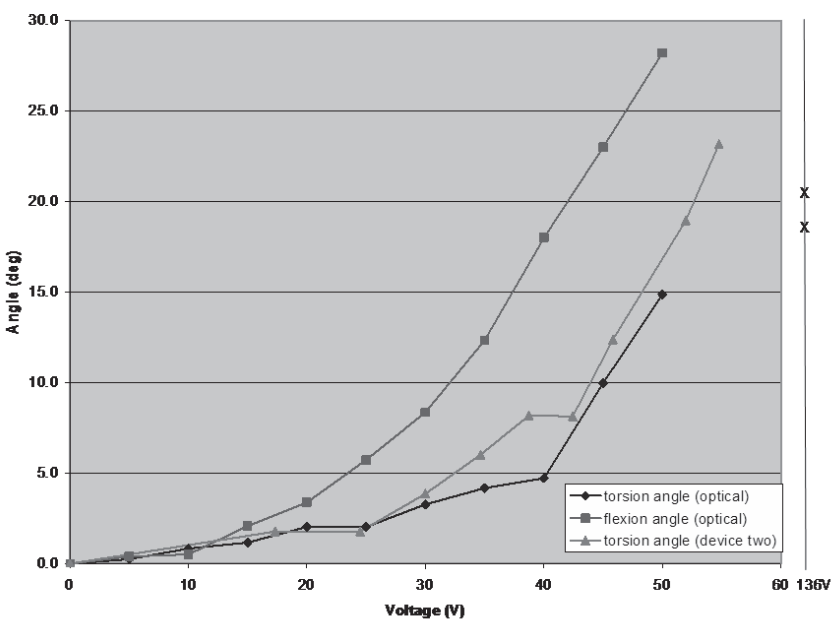

Figure 8: Experimental test data. For comparison, the best quadrant of operation of the highest-performing large tip/tilt mirror is shown (X's at 136V) from [1]. This rotation stage achieves larger static deflection angles at lower voltages.

Since the current process is not ideal for creating mirror face sheets, an assembled face sheet shown in (fig 6) is used which serves merely as an assembly technology demonstrator. This face sheet was affixed to the mirror mount of the two-axis rotation stage using epoxy. A low inertia mirror can be fabricated by sacrificing some process complexity as demonstrated in $[3,12]$. Fabrication of such a mirror would require at least one additional mask. The mirror face sheet and mechanisms can be built in different processes and optimized separately without requiring the 
compromise in performance or increase in process complexity that monolithic integration would require.

\section{CONCLUSIONS}

Microassembly provides an attractive option for fabricating complex MEMS structures using a simple fabrication process. This simplicity translates into fast design cycles and high fabrication yield. Microassembly yield increases with the improved tooling presented in this paper: tethers that break gently and predictably, grippers that rotate parts by exactly $90^{\circ}$, and robust sockets. This paper demonstrates that in addition to the speed and yield benefits, a single-mask microassembly-based process can be used to create high performance devices. The electrostatically-actuated tip/tilt micromirror described here sets a new record for DC deflection angle in one of the two axes of rotation.

\section{REFERENCES}

[1] Milanovic V., D.T. McCormick, and G.A. Matus, Gimbal-less Monolithic Silicon Actuators for Tip-Tilt-Piston Micromirror Applications. IEEE Journal of Select Topics in Quantum Electronics, 2004. 10(3): p. 462-471.

[2] Hollar S., A. Flynn, C. Bellew, and K.S.J. Pister. "Solar powered $10 \mathrm{mg}$ silicon robot". Proceedings IEEE Sixteenth Annual International Conference on Micro Electro Mechanical Systems. Kyoto, Japan. 19-23 Jan. 2003. (2003)

[3] Milanovic V., M. Last, and K.S.J. Pister, Laterally actuated torsional micromirrors for large static deflection. IEEE Photonics Technology Letters, 2003. 15(2): p. 245-7.

[4] Cohn M.B., Assembly Techniques for Microelectromechanical Systems, in Engineering -- Electrical Engineering and Computer Sciences. 1997, University of California, Berkeley: Berkeley. p. 150.

[5] Guckel H., T. Christenson, and K. Skrobis, Metal micromechanisms via deep X-ray lithography, electroplating and assembly. Journal of Micromechanics \& Microengineering, 1992. 2(4): p. 225-8.

[6] Fischer K. and H. Guckel, Long throw linear magnetic actuators stackable to one millimeter of structural height. Microsystem Technologies, 1998. 4(4): p. 180-3.

[7] Dechev N., W.L. Cleghorn, and J.K. Mills. "Tether and joint design for microcomponents used in microassembly of $3 \mathrm{D}$ microstructures". MEMS/MOEMS Components and their Applications. San Jose, CA,USA. 26-27 Jan. 2004. (2004)

[8] Tsui K., A.A. Geisberger, M. Ellis, and G.D. Skidmore, Micromachined end-effector and techniques for directed MEMS assembly. Journal of Micromechanics \& Microengineering, 2004. 14(4): p. 542-9.

[9] Saini R., A. Geisberger, K. Tsui, C. Nistorica, M. Ellis, and G. Skidmore. "Assembled MEMS VOA". 2003 IEEE/LEOS International Conference on Optical MEMS. Waikoloa, HI. 18-21 Aug., (2003)
[10] Last M., V. Subramaniam, and K.S.J. Pister. "Out of plane motion of assembled microstructures using a single-mask SOI process". TRANSDUCERS '05. The 13th International Conference on Solid-State Sensors, Actuators and Microsystems. Digest of Technical Papers. Seoul, South Korea, 5-9 June 2005., Korean Sensors Society. (2005)

[11] Last M., Silicon on Insulator Pick and Place Microassembly, in Engineering - Electrical Engineering and Computer Science. 2005, University of California, Berkeley: Berkeley. p. 168. 